\title{
Conceptual design of CFETR ECRH equatorial launcher and upper launcher
}

\author{
Yunying Tang ${ }^{1, *}$, Fukun $\mathrm{Liu}^{1}$, Xiaojie $\mathrm{Wang}^{1}$, Wei $\mathrm{Wei}^{2}$, Liyuan Zhang ${ }^{1}$, Handong $\mathrm{Xu}^{1}$, Dajun $\mathrm{Wu}^{1}$, Weiye $\mathrm{Xu}^{1}$,and Jian \\ Wang ${ }^{1}$ \\ ${ }^{1}$ Institute of Plasma Physics, Chinese Academy of Sciences, Shushanhu Road 350, Hefei 230031, Anhui, China \\ ${ }^{2}$ Hefei University of Technology, Tunxi Road 193, Hefei 230009, Anhui, China
}

\begin{abstract}
The Electron Cyclotron Resonance Heating (ECRH) system of China Fusion Engineering Test Reactor (CFETR) is designed to inject $20 \mathrm{MW}$ RF power into the plasma for heating and current drive (H\&CD) applications. The ECRH system consists of 20 gyrotrons, the associated power supplies, the transmission lines and one launcher. In order to compare the launcher performance from equatorial and upper ports, two types of launcher are designed in this paper. In equatorial launcher (EL), twenty in-vessel transmission lines are divided into four groups. Every five gaussian beams from in-vessel transmission lines face one fixed focusing mirror and one steering mirror. All gaussian beams are injected into the plasma with optimal toroidal and poloidal angles calculated by C3PO/LUKE code. In upper launcher (UL), twenty-one transmission lines are supposed and divides into three groups. Every seven gaussian beams are injected into one fixed focusing mirrors and all twenty-one beams reflected to one common steering mirror finally. The optical transmission characteristics and the convergence information of gaussian beams are checked and optimized. The EL or UL is installed on the equatorial or upper port with a port-plug modular structure.
\end{abstract}

\section{Introduction}

The objective for International Thermonuclear Experimental Reactor (ITER) [1] is to demonstrate the scientific and technological feasibility of applying fusion energy for peaceful purposes. Based on the existing physics and technology of ITER, CFETR works on the technology which cannot be completed by ITER. The mission and goal of CFETR are as follows: (1) Fusion power 200-1500 MW; (2) Duty cycle time (or burning time) $\geqq 50 \%$; (3) Tritium should be self-sufficiency by blanket, TBR (Tritium Breeding Ratio) $\geqq 1$. It is challenged by integrated design considering all crucial elements [2,3], which include integrated plasma scenarios for steady-state operation and engineering optimization, particularly inboard radial space for tritium breeding modules and the tradeoff of plasma faced surface for tritium breeding and heating and current drive ( $\mathrm{H}$ and $\mathrm{CD}$ ), diagnostics, burning ratio of fuelled particles, plasma burning duty factor, $\mathrm{RH}$, and so on.

To assist and sustain CFETR operation in various scenarios, basing on the design experience of EAST ECH system [4,5], an ECH\&ECCD system [6,7] operating at $170 \mathrm{GHz}, 20 \mathrm{MW}$ power continuous wave (CW) will be installed for the CFETR with the power injected via two types of launchers, the equatorial launcher and the upper launcher.

In this paper, central heating and current drive are the main physical requirements for EC system. Because of the excellent local power deposition and simple coupling between wave and plasma, megawatt steady-state ECH system is in the first priority to be considered among all the auxiliary heating systems. ECCD maintains the non-inductive current drive and controls the current profile during CFETR steady-state operation.

The main purpose of this paper is to present the status of our current design work. The structure of ECH system is introduced in section 2. The designs of equatorial launcher and upper launcher designed basing on the initial small-size CFETR device [2,3] are described in section 3 and 4. Finally, the conclusion is given.

\section{ECH system}

ECH system is composed of microwave subsystem, high voltage power supply (HVPS) subsystem, interlock and control protection subsystem, vacuum pumping subsystem and water-cooling subsystem (Fig.1). The microwave subsystem consists of microwave source, transmission line and EC launcher, which show the generating, transferring and injecting process of microwave power respectively. HVPS which is mainly formed by cathode power supply and body power supply is in charge of power supply for microwave source. Each cathode power supply provides two gyrotrons [8-11] PSM structure is chosen in the cathode power supply. It adopts all-controlling switching elements and the switching speed is very quick. Therefore the independent 
crow-bar protection for gyrotron do not need here. Independent body power supply, superconducting magnet power supply, filament power supply, gun coil power supply, ion pump power supply and collector coil power supply are configured to every gyrotron. Interlock and control protection subsystem, water-cooling subsystem and vacuum subsystem assure the safe and reliable operation of the overall $\mathrm{ECH}$ system. Interlock and control protection subsystem commands all the subsystems operating according to the setting procedures and parameters and monitors all kinds of operating parameters in real time. Once error occurs in the system, interlock and control protection subsystem will execute protective action immediately. Water-cooling subsystem cools all the microwave devices which are needed to be cooled in the ECH system. Vacuum subsystem ensures superconducting magnets, matching optics unit (MOU) and corrugated waveguides running in a good vacuum state.

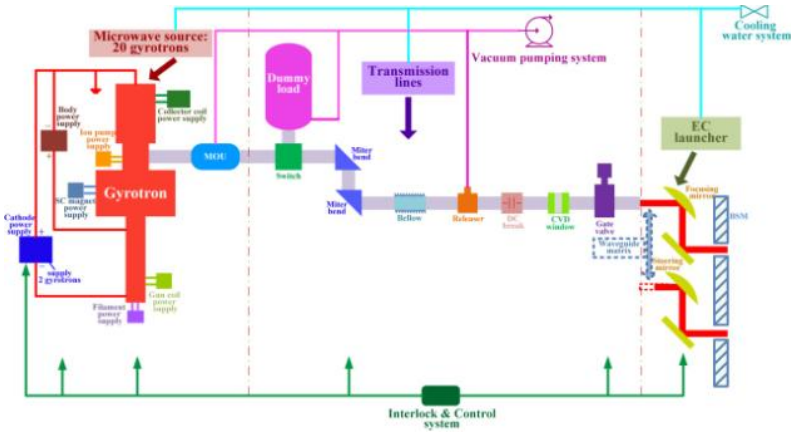

Fig. 1. The constitution of CFETR ECH system. It contains microwave sources, transmission lines, EC launcher, cooling water subsystem, vacuum pumping subsystem, interlock \& control subsystem and HVPS.

In ECCD, the resonance condition for wave-particle in velocity space is given by the following equation [12]:

$$
\omega=n \omega_{c e} / \gamma+k_{/ /} v_{/ /}
$$

where $\omega$ is the wave frequency, $\omega_{c e}$ is the electron cyclotron frequency, $n$ is the electron harmonic number, $\gamma=1 / \sqrt{1-\left(v^{2} / c^{2}\right)}$ is the relativistic form-factor, which is a consequence of the relativistic correction of the electron mass, $c$ is the velocity of light in vacuum. The $k_{/ / N / /}$ term is the Doppler effect, which plays an important role in ECCD and causes both shift and broadening of the absorption line, where $v_{/ /}, k_{/ /}$are the parallel components of the velocity of the resonant electrons and wave vector of the EC wave, respectively. In ECH system, two modes can be used to heat the plasma: $\mathrm{O}$ mode and $\mathrm{X}$ mode [13]. If the EC wave is injected into plasma from low field side, only $\mathrm{O}$ mode and $\mathrm{X} 2$ mode can be adopted. The probable resonance layers and heating methods in CFETR ECCD system are shown in Fig. 2. Taking the frequencies adopted in the present tokamaks into consideration, $110 \mathrm{GHz}, 140 \mathrm{GHz}, 170 \mathrm{GHz}$ and $230 \mathrm{GHz}$ are chosen in the analysis. In Fig. 2(a), when the center toroidal field is $5 \mathrm{~T}, 140 \mathrm{GHz}, 170 \mathrm{GHz}$ and $230 \mathrm{GHz}$ satisfy the ECCD system demand. When the center toroidal field of CFETR is up to $7 \mathrm{~T}$ in the future shown in Fig. 2(b), only $170 \mathrm{GHz}$ and $230 \mathrm{GHz}$ can be accepted. Combining with the mature manufacture technology of ITER $170 \mathrm{GHz}$ gyrotron, $170 \mathrm{GHz}$ is chosen as working frequency of ECH system in CFETR according equation (1) and EC wave would be coupled to plasma with $\mathrm{O} 1$ mode.
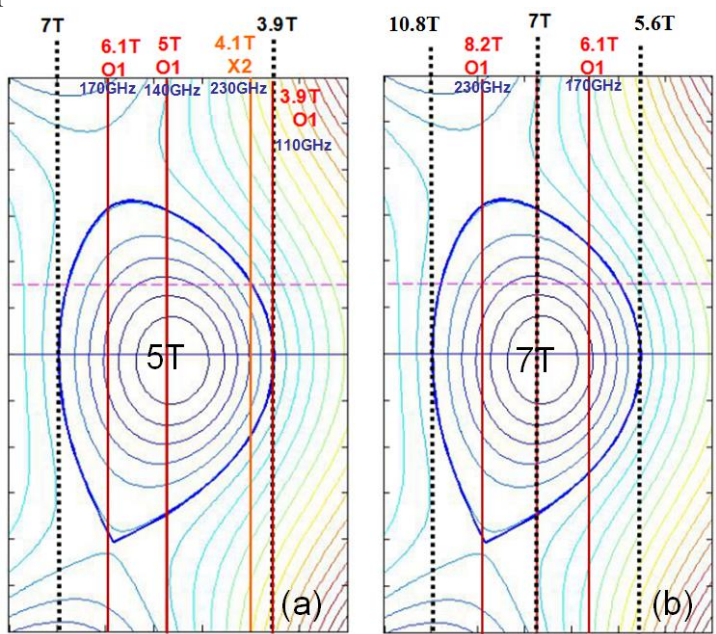

Fig. 2. (a) The center toroidal field $B_{T}=5 T$; (b) $B_{T}=7 T$.

The microwave subsystem is made up of RF source, transmissions line and launcher. The design of launcher is similarly ITER EL and UL[14-17], two types of launchers are designed. In Fig. 3, it shows ECH system for CFETR where the injected launcher is EL for instance. RF sources contain twenty $170 \mathrm{GHz} / 1 \mathrm{MW}$ gyrotrons which take charge of generating microwave power. $20 \mathrm{MW}$ microwave power is transferred by 20 transmission lines and injected into the plasma via EL. Transmission lines should satisfy a series of technical requirements, therefore there are different microwave components distributed along the line, such as MOU, corrugated waveguide, miter bends and some auxiliary equipments (Fig. 4). The diameter of corrugated waveguide is $63.5 \mathrm{~mm}$ and the transferring mode in corrugated waveguide is $\mathrm{HE}_{11}$ mode. The miter bends in the transmission lines possess many functions, such as changing microwave power transferring direction, measuring the power [18], monitoring the arc and adjusting the polarization information [19]. The auxiliary equipments contain direct current (DC) break, vacuum pumping sections, chemical vapor deposition (CVD) window, gate valve and so on [20-22]. The DC break separates electrical potential along the transmission line to eliminate unnecessary electrical closed loops. The pumping section evacuates the transmission line through a gap of the waveguide and the whole transmission lines are evacuated to $\leq 2 \times 10^{-3} \mathrm{~Pa}$ in order to reliably transmit $1 \mathrm{MW}$ RF wave. In each transmission line, there is one CVD window located close to the launcher in order to separate the high vacuum of CFETR vessel from vacuum transmission line. Gate valve is located at the end of transmission line and it is shut off immediately when one discharge is over.

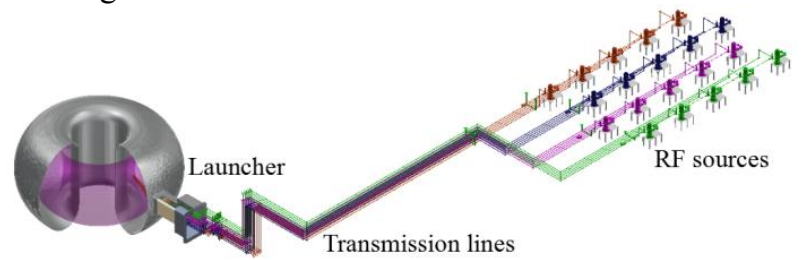

Fig. 3. ECH system for CFETR. 


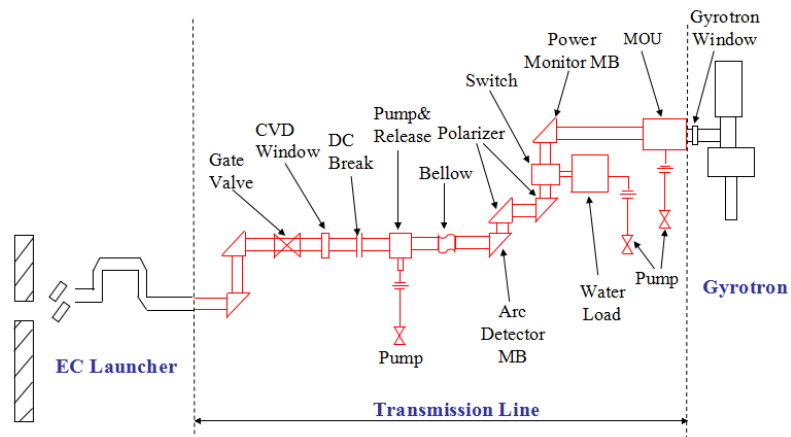

Fig. 4. Schematic of a generic TL.

\section{Equatorial launcher}

The wave with the specific mode, polarization information and incident angle is injected into CFETR plasma by the quasi-optical launcher which should be located on the special position for the optimal heating and driving current.

The EL is installed on the equatorial port with sectional area $2200 \mathrm{~mm} \times 1750 \mathrm{~mm}$. Total $20 \mathrm{MW}$ microwave power from 20 gyrotrons should be injected into plasma via one EL. Before designing the EL, the injecting position of the microwave power is decided by C3PO/LUKE code [23] via calculating the driven current in different positions. In $\mathrm{C} 3 \mathrm{PO} / \mathrm{LUKE}$ code, the injection angles of the beams are defined as Fig. 5. $\theta$ is the poloidal injection angle and $\varphi$ is the toroidal injection angle. The distance from the center of CFETR horizontal plane to the injection mirror in vertical plane is marked as $\mathrm{Za}$ in Fig. 6(a). The distance from the middle point between injection mirror and focusing mirror to the CFETR center in horizontal plane can be seen in Fig. 6(b) and is named $\mathrm{Ra}$. The distance in horizontal plane between injection mirror and focusing mirror is $100 \mathrm{~cm}$.
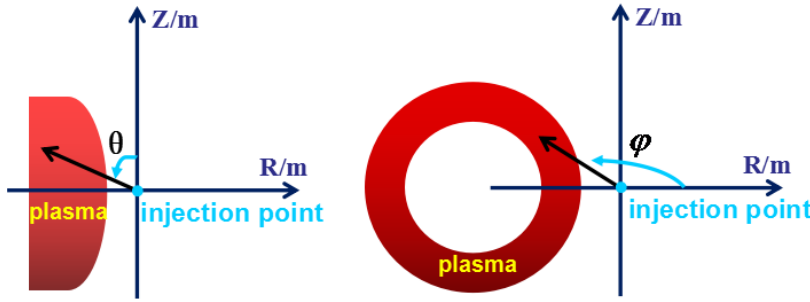

Fig. 5. Definition of the injection angles ( $\theta$ : poloidal injection angle; $\varphi$ : toroidal injection angle).

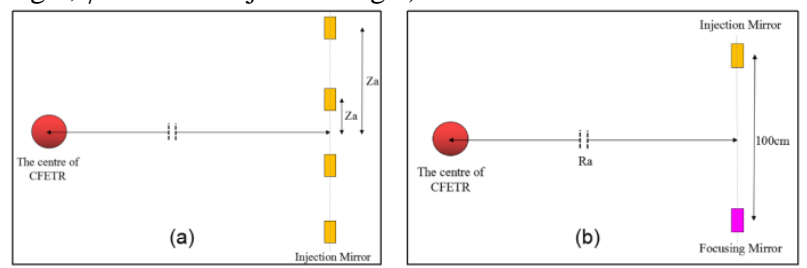

Fig. 6. The injection positions of the injection mirrors and the focusing mirrors.

With the profiles of $T_{e}$ and $n_{e}$ as shown in Fig. 7, the driven current is optimized with the different positions of injection mirrors and focusing mirrors. Combining the size and shape of the CFETR equatorial port, $\mathrm{Ra}$ is chosen as $1000 \mathrm{~cm}$ and $\mathrm{Za}$ is $\pm 22.5 \mathrm{~cm}$ or $\pm 67.5 \mathrm{~cm}$ (" + " is higher than the center of CFETR horizontal plane and "-" is lower than the center of CFETR horizontal plane). The calculating configuration is ITER-like configuration which is not symmetrical about the center horizontal plane of CFETR, so the different driven current of four height positions of injection mirrors ((a)-67.5mm, (b) $-22.5 \mathrm{~mm}$, (c) $22.5 \mathrm{~mm}$, (d) $67.5 \mathrm{~mm}$ ) are given in Fig. 8 . The driven currents are gotten with toroidal angles and poloidal angles changing in a certain range. The maximum driven currents in four different positions are all about $29 \mathrm{kA}$ when the injecting power is $1 \mathrm{MW}$. The optimal toroidal injecting angles are all about $206^{\circ}$ and the optimal poloidal injecting angles are about $83^{\circ}, 88^{\circ}$, $94^{\circ}$ and $98^{\circ}$ respectively.

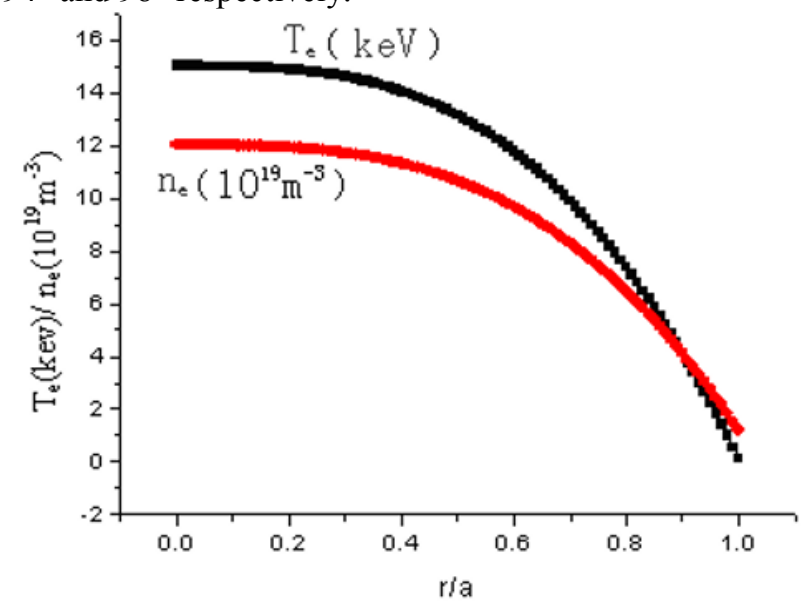

Fig. 7. The profiles of $T_{e}$ and $n_{e}$.

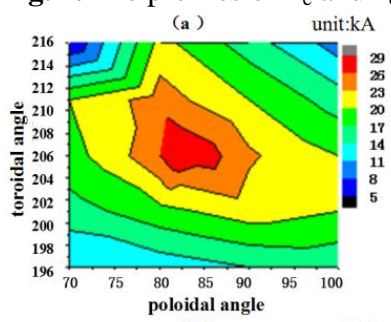

(b)
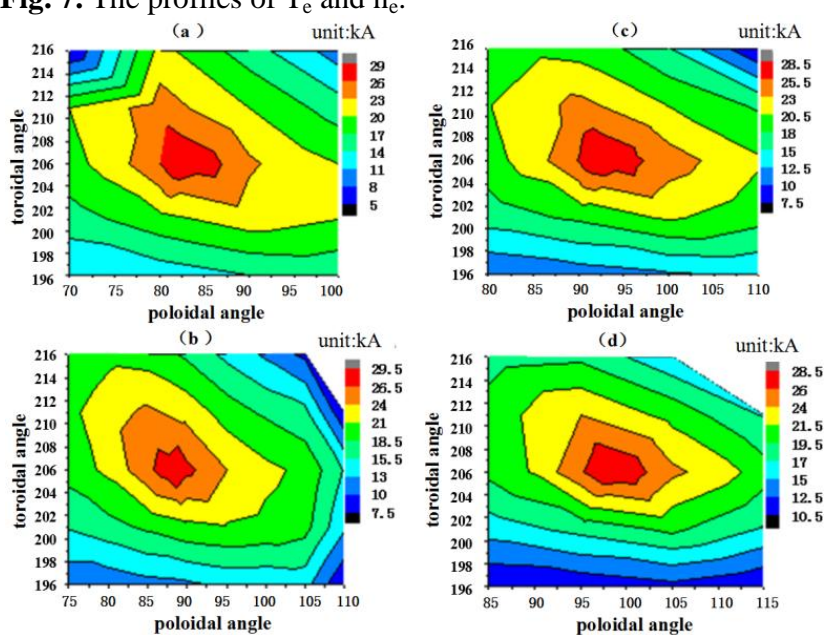

Fig. 8. In four heights ((a)-67.5mm, (b)-22.5mm, (c) $22.5 \mathrm{~mm}$, (d) $67.5 \mathrm{~mm}$ ) of the injection mirrors, the driven current vs different toroidal angles and poloidal angles.

EL is made up of in-vessel transmission lines (in-vessel transmission lines and ex-vessel transmission lines are separated by external surface of the flange), vacuum shielding, internal shield, focusing mirror, steering mirror, drive mechanisms of steering mirror, Blanket Shielding Module (BSM) [24], flange and so on (Fig. 9 and Fig. 10). The EL is installed on the CFETR equatorial port with port-plug modular structure. The in-vessel transmission lines are extended from transmission lines, restrain the microwave power and transfer the power with certain route. The optical mirrors contain focusing mirrors and steering mirrors which are in charge of changing the transferring route of gaussian beams. The vacuum shielding separates the high vacuum of CFETR chamber from external environment. One side 
of vacuum shielding connecting with tokamak has the same vacuum with the vacuum chamber. The other side is in the atmosphere facilitating the installing and repairing microwave devices, and controlling electric motors which are the driving mechanisms of steering mirrors. The internal shield and BSM will both shield the neutron radiation in the physics experiments in the future. The flange takes charge of the installation and jointing between EL and port.

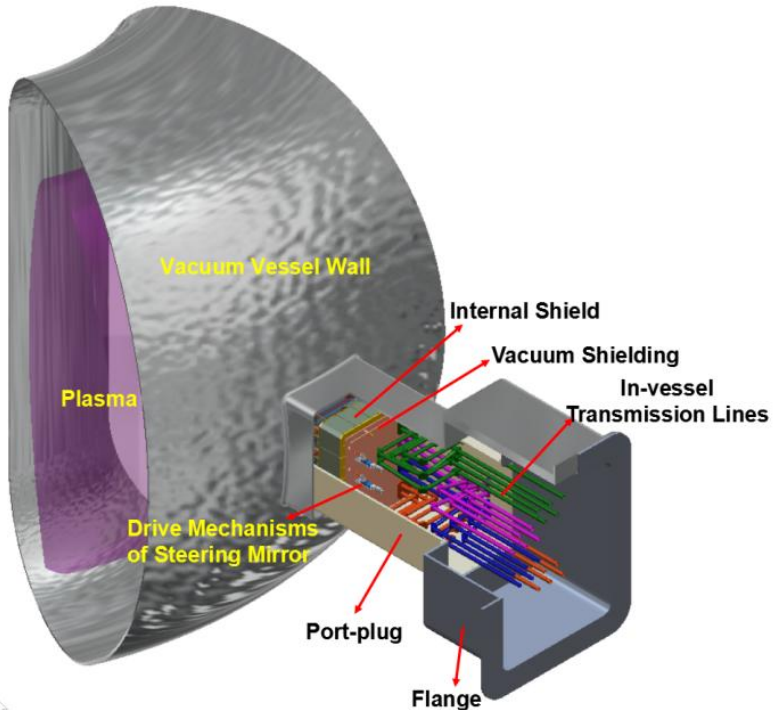

Fig. 9. The overall installation of the equatorial launcher.

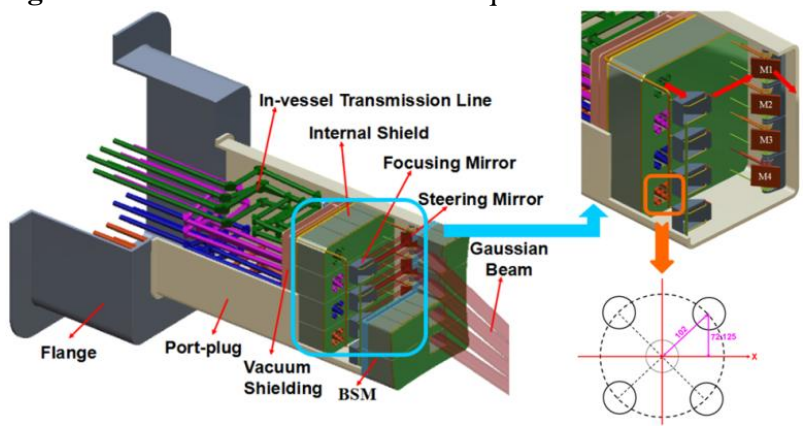

Fig. 10. The internal structure of equatorial launcher and the transferring route of gaussian beams.

Twenty in-vessel transmission lines are divided into four groups in EL and each group has five transmission lines. The top and the bottom of groups are arranged by four miter bends in every transmission line and the middle two groups are arranged by two miter bends. Miter bends increase the transmission loss, however, in order to converge the power from transmission lines into focusing mirrors, these are the integrant minimum quantities of bends. There are four focusing mirrors and four steering mirrors in ECH system. Five waveguide ends of the in-vessel transmission lines in each group face one fixed focusing mirror and five gaussian beams from the waveguide ends are injected into this focusing mirror, reflected to one steering mirror and then injected into the plasma of CFETR finally. The optical transmission routes are shown in the top right of Fig. 10. Four steering mirrors are fixed in poloidal direction and can steer in toroidal direction within a certain range of angles. The waveguide ends of each group are shown in the bottom right of Fig. 10. Four waveguide ends arrange around the middle end and they distribute symmetrically about the center. The distance between every ambient waveguide end and the center waveguide end is $102 \mathrm{~mm}$. The detailed designs of focusing and steering mirrors and the optical transmission characteristics of gaussian beams are optimized in Fig. 11. The distance from waveguide end to focusing mirror, from focusing mirror to steering mirror, and from steering mirror to plasma center are $500 \mathrm{~mm}, 1000 \mathrm{~mm}$ and $4300 \mathrm{~mm}$, respectively. When the focal length of focusing mirror is $7500 \mathrm{~mm}$, five gaussian beams are converged in plasma center shown in Fig. 11(a). The power density distributed in plasma center is shown in Fig. 11(b) with maximum power density $126.4 \mathrm{MW} / \mathrm{m}^{2}$.

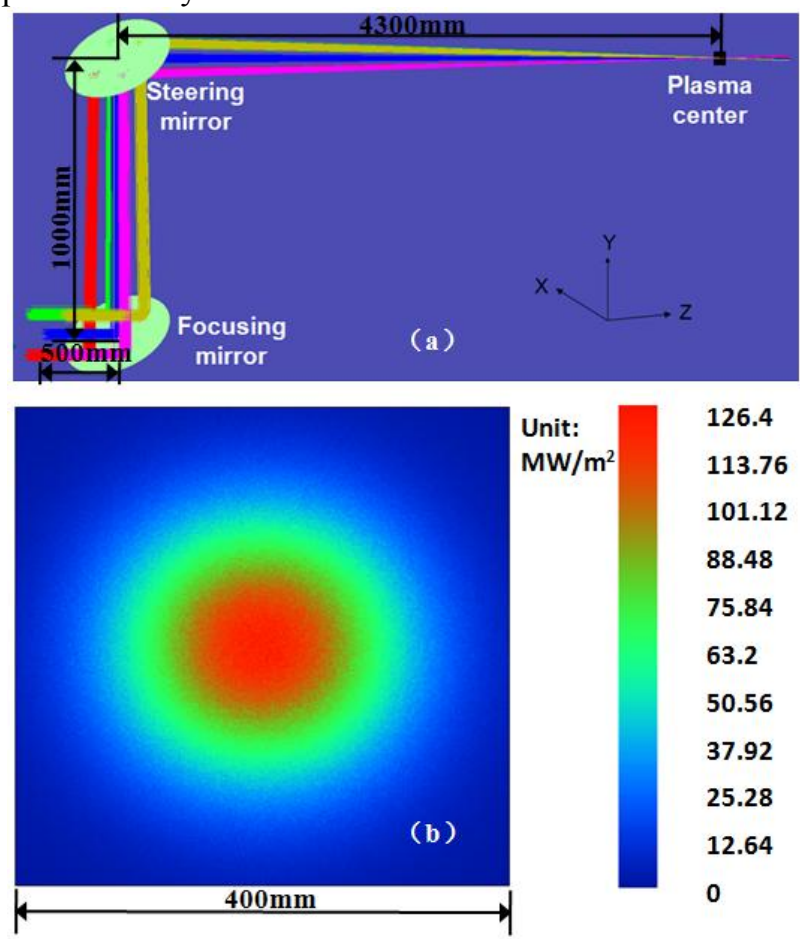

Fig. 11. The transferring process of five gaussian beams. (a)the transmission trace of gaussian beams; (b)the distribution of power density in plasma center.

\section{Upper launcher}

There are only eight equatorial ports in CFETR and maybe none of them will be allocated to the ECH system. In that case, whether the microwave power can be injected from upper port is very important and should be proved. The driven current is also calculated by $\mathrm{C} 3 \mathrm{PO} / \mathrm{LUKE}$ code.

The upper port is narrow and long compared with the equatorial port (Fig.12). The length of upper port in vertical direction is $13.29 \mathrm{~m}$ and the horizontal cross-section of upper port is similar to a trapezoid whose wide length and narrow length are about $1772 \mathrm{~mm}$ and $416 \mathrm{~mm}$ respectively and whose height is about $3609 \mathrm{~mm}$. The narrow and long shape and irregular cross-section of the upper port bring great challenge to design the UL in the ECH system. 


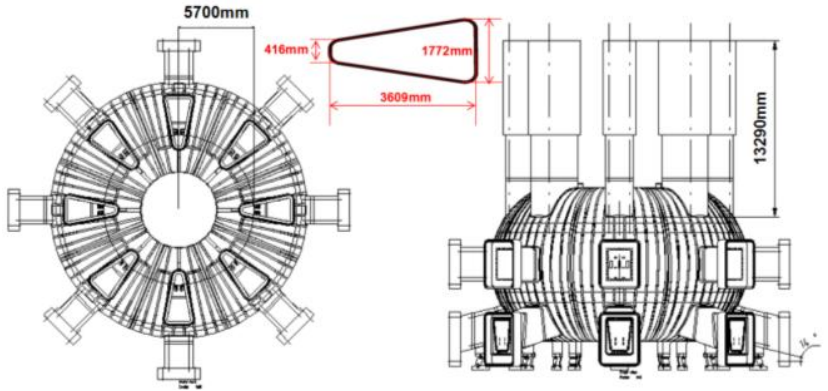

Fig. 12. The shape and size of the upper port.

On account of the special shape and size of the upper port, the injection position of the UL is pre-estimated before physics calculation. The horizontal distance Ra and the vertical distance $\mathrm{Za}$ (defined same as Fig. 6) are chosen $7.919 \mathrm{~m}$ and $4.787 \mathrm{~m}$, respectively. The driven current is calculated and optimized in Fig. 13. The total driven current is $1.46 \mathrm{MA}$ and the coverage area of the current is about $\rho \sim 0.1-0.8$ when $18 \mathrm{MW}$ microwave power is supposed to inject into the plasma. The adjustable ranges of the toroidal angles and poloidal angles are $\varphi \sim 210^{\circ}-230^{\circ}$ and $\theta \sim 135^{\circ}-145^{\circ}$.
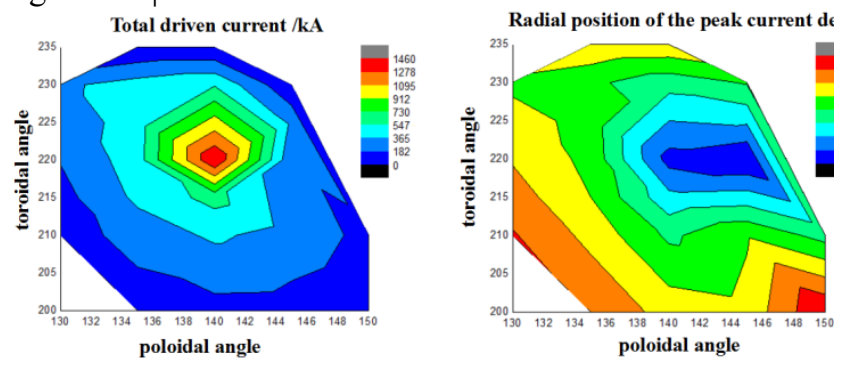

Fig. 13. Total driven current and radial position of the peak current density vs toroidal angles and poloidal angles.

During the UL design, twenty-one gyrotrons and transmission lines are supposed. The arrangement principle of the RF sources and transmissions lines is similar to the Fig. 3 and not given here. The UL forms one port-plug modular structure made up of multiple microwave transmission components. It contains twenty-one in-vessel corrugated waveguides, miter bends, optical mirrors and so on. Twenty-one gaussian beams from in-vessel transmission lines are divided into three groups, injected to three fixed focusing mirrors, reflected from one common steering mirror and injected into the plasma at last. The arrangement of twenty-one transmission lines located in the flange is shown in the top left of Fig.14. The distance between top and bottom groups is $400 \mathrm{~mm}$ and the left group goes to the center of top and bottom groups is $350 \mathrm{~mm}$. In each group, ambient six transmission lines distribute surrounding one center transmission line equably and the distance between every two neighboring transmission lines is $100 \mathrm{~mm}$. From the right of Fig.14, the port-plug modular structure is composed of three sections which will bring the convenience of hoisting and installation in the future engineering construction. The upper section is mainly flange and twenty-one in-vessel transmission lines. The flange is in charge of the docking duty with the upper port. The middle section is constituted by the vacuum shielding, corrugated waveguides and miter bends. The bottom section primarily contains optical mirrors as well as their affiliated structure. Every seven gaussian beams from each group of transmission lines inject into the correspondent focusing mirror. The design of the optical mirrors and the transmission characteristics of gaussian beams are also optimized and the power distributions of all beams deposited in steering mirror and plasma center are shown in Fig. 15. The focal length of focusing mirror is $10 \mathrm{~m}$.

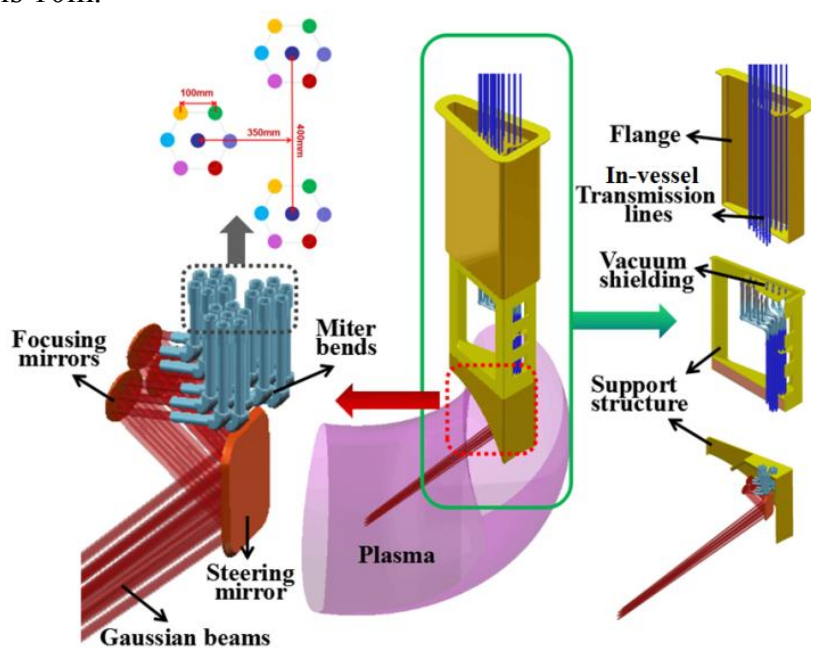

Fig. 14. The design structure of the upper launcher.

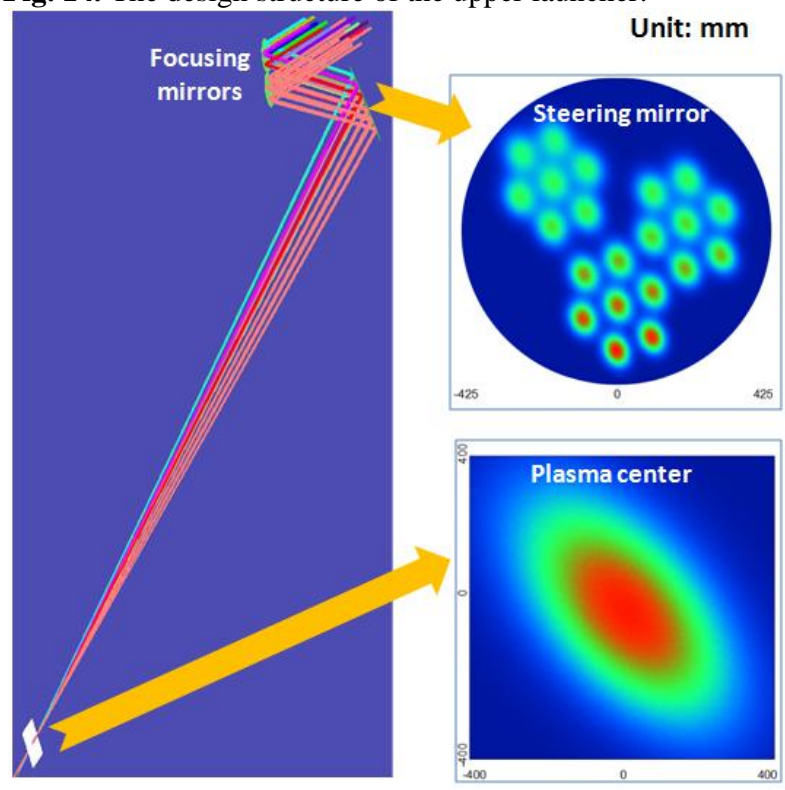

Fig. 15. The transmission characteristics of gaussian beams is analyzed. All guassian beams are reflecting to the steering mirror whose radius is $425 \mathrm{~mm}$ and injected into plasma with spot length $800 \mathrm{~mm}$.

The UL is installed on the upper port as shown in Fig. 16. Total gaussian beams are reflected from the steering mirror, transfer through BSM and then are injected into plasma. From above physics calculation, the optimal toroidal angle is $220^{\circ}$, which means the total beams will steer $40^{\circ}$ in toroidal direction. Oversize steering angle in toroidal direction is dangerous, because the powerful beams will be likely to burn the port edge. In order to prevent this case happening, the steering mirror is not symmetrical as three focusing mirrors and is moved $150 \mathrm{~mm}$ towards the left in the opposite direction of the 
gaussian beams. The UL design is relatively simple so far. More detailed design and analysis will be carried on.

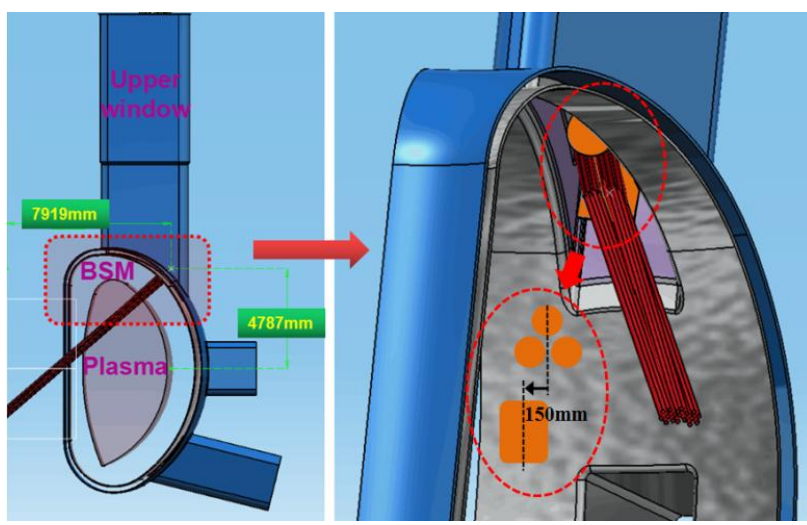

Fig. 16. The installation effect of the upper launcher.

\section{Conclusions}

According to the port position of CFETR tokamak, two types of injection launcher-EL and UL are designed with quasi-optics transferring pattern. The launcher is installed on equatorial port or upper port with port-plug modular structure. In the detailed research of the EL and $\mathrm{UL}$, the injecting position and injecting angles of steering mirror are calculated by $\mathrm{C} 3 \mathrm{PO} / \mathrm{LUKE}$ code. Then according to the transmission characteristic of gaussian beams, the transmission routes and converging of all the wave beams are optimized by optical simulation software. After optimizing, the deploy of transmission lines in the launcher and the information of the optical mirrors are gotten. At last, the overall design of the EL and UL, including focusing mirror, steering mirror, driving structure of the steering mirror, in-vessel transmission lines, blanket shield module, internal shield module, vacuum shielding, supporting structure, flange and so on, are all explored.

Compared with two launchers, each launcher has its advantages and disadvantages. On the driven current, UL can get about $81 \mathrm{kA}$ driven current when $1 \mathrm{MW}$ microwave power is injected into the plasma, while EL can only get $29 \mathrm{kA}$. The driven current of UL is better than the EL. On the engineering design, because of the major size and regular shape of the equatorial port, the layout of the microwave components in the EL is not as compact as the UL and more than one steering mirrors can be adopted in the EL. The difficulty on EL engineering design is lower than UL.

The present design of EL and UL is based on the initial small size CFETR, more research and design of ECRH launchers with respect to the present CFETR parameters are on-going.

\section{Acknowledgement}

This work has been supported by the National Key R\&D Program of China under Grant No. 2017YFE0300200 and the Strategic Priority Research Program of Chinese Academy of Sciences under Grant No. XDA14040500.

\section{References}

1. R. Aymar, P. Barabaschi and Y. Shimomura, Plasma Phys. Control. Fusion 44, 5, 519-565 (2002)

2. Y. T. Song et al., IEEE Trans. Plasma Sci. 42, 3, 503-509 (2014)

3. B. N. Wan et al., IEEE Trans. Plasma Sci. 42, 3, 495-502 (2014)

4. X. J. Wang et al., Fusion Eng. Des. 96-97, 181-186 (2015)

5. H. D. XU et al., Plasma Sci. Technol. 18, 4, 442-448 (2016)

6. Jiangang Li, et al., EPJ Web Conf. 149, 01011 (2017)

7. Y. Y. Tang et al., Fusion Eng. Des. 94, 48-53 (2015)

8. K. Sakamoto et al., Nucl. Fusion 43, 729-737 (2003)

9. P. C. Kalaria, M. V. Kartikeyan and M. Thumm, IEEE Trans. Plasma Sci. 42, 1522-1528 (2014)

10. R. K. Dhakad, G. S. Baghel, M. V. Kartikeyan and M. K. Thumm, IEEE Trans. Plasma Sci. 43, 391-397 (2015)

11. B. Piosczyk et al., IEEE Trans. Plasma Sci. 32, 413-417 (2003)

12. W. Wei et al., Chin. Phys. B 23, 5 (2014)

13. W. Wei et al., Nuclear Fusion and Plasma Physics, 33, 2, 147-154 (2013)

14. T. Omori, et al., Fusion Eng. Des. 86, 951-954 (2011)

15. K. Takahashi, et al., AIP Conference Proceedings 1580, 546-549 (2014)

16. K. Kajiwara, et al., Fusion Eng. Des. 89, 6-10 (2014)

17. D. Strauss, et al., Fusion Eng. Des. 89, 1669-1673 (2014)

18. D. J. Wu et al., IEEE Access, 5, 6187-6191 ( 2017)

19. D. J. Wu et al., J. Fusion Energy, 33, 634-639 (2014)

20. R. A. Olstad, J. L. Doane and C. P. Moeller, Fusion Eng. Des. 74, 331-335 (2005)

21. R. W. Callis et al., Fusion Eng. Des. 84, 526-529 (2009)

22. F. Gandini et al., Fusion Sci. Technol. 59, 709-717 (2011)

23. W. Wei et al., Chin. Phys. B 25, 15201 (2016)

24. P. Spaeh et al., Fusion Eng. Des. 89, 960-964 (2014) 